\title{
LXXXV. On the currents produced by the actuation or induction of instantaneous electric currents
}

\section{Professor Stefano Marianini}

To cite this article: Professor Stefano Marianini (1842) LXXXV. On the currents produced by the actuation or induction of instantaneous electric currents, Philosophical Magazine Series 3, 21:141, 497-509, DOI: $10.1080 / 14786444208650491$

To link to this article: http://dx.doi.org/10.1080/14786444208650491

Published online: 01 Jun 2009.

Submit your article to this journal $₫$

Џ Article views: 2

Q View related articles $\sqsubset$ 
LONDON, EDINBURGH AND DUBLIN

\section{PHILOSOPHICAL MAGAZINE \\ AND \\ JOURNAL OF SCIENCE.}

SUPPLEMENT to VOL. XXI. THIRD SERIES.

LXXXV. On the Currents produced by the Actuation or Induction of instantaneous Electric Currents. By Professor Stefano Marianini*.

Currents produced by the Leyden-electrical Induction.

I. THE facility with which, by means of the instruments

described in the preceding memoirt, the presence of an instantaneous electric current in a metallic wire is detected, encouraged me to seek indications also of the currents which are derived from actuation or induction.

I had surrounded a little cylinder of iron with two copper wires covered with silk, which formed two parallel coils, and having placed the cylinder upon the cap of a needle, I made use of this apparatus as a re-electrometer, the delicacy of which varied as I made the currents pass through one only of the said coils, or through both at the same time, or joined them so that the current had to pass through one first, and then through the other; with these two parallel coils I made my first attempt on the Leyden-electrical induction. Having however taken that re-electrometric cylinder from the cap of the needle, I connected one of the wires which surrounded it with the ends of the coil of a re-electrometer, and the Leyden jar being discharged upon the other wire, connecting its extremities with the two coatings, I have seen the needle of the instrument deviate a similar number of degrees.

* Translated from Memorie di Fisica Sperimentale scritte dal Professore Stefano Marianini dopo il 1836. Anno Primo, 1837. Modena, 1838. It was from the Anno Secundo, 1838, of this work that the memoir by the author inserted in Phil. Mag. S. 3. vol. xviii. p. 193 was translated. Prof. Henry's researches on the induced currents of common electricity, to which the memoir now given relates, will be found in Phil. Mag. S. 3 . vol. xvi. p. 551. The experiments of these philosophers were we believe quite independent, and must have been nearly contemporaneous; but the priority is probably due to the Italian.-EDIr.

[ $t$ The preceding memoir here referred to relates to an instrument for measuring the force both of instantaneous and non-instantaneous electric currents.-EDIr.]

Phil. Mag. S. 3. No. 141. Suppl. Vol. $21 . \quad 2$ L 
I suspected that the deviations obtained in this and other similar experiments might proceed, not from induction occasioned immediately by the instantaneous current of the jar, but from magneto-electric induction, produced by the temporary magnetization of the iron cylinder surrounded by the two coils. However, the cylinder being taken away, and a small glass rod substituted, the same phænomenon took place.

II. As I made use of a jar highly charged and the wires presented some metallic points uncovered, it was probable that part of the current might make itself a passage from one wire to the other, and that the effects observed might proceed from a real current transferred into the wire of the re-electrometer, and not from a movement of the electricity of the wire itself excited by the current which was made to pass near it.

A coil of large uncovered copper wire was enveloped in four layers of silk; I then inclosed it in sixty coils of fine copper wire covered with silk; with this apparatus I made many trials, and I observed,-1 st, that with the smail jar (having little more than half a square decimeter of conting) the needle always deviated from the part, whence it also deviated when I discharged the jar itself, and in the same manner upon the wire of the re-electrometer, as much as when I discharged it upon the coil of uncovered copper wire (keeping tha fine wire in communication with that of the re-electrometer), as when I put the coil of uncovered copper wire in communication with this, and discharged the jar upon the fine wire; $2 \mathrm{nd}$, that with the large jars (nineteen square decimeters of coating), if they were highly charged, the needle deviated in the same manner as when they were discharged upon the re-electrometric wire; but if they were slightly charged the deviation was different.

III. I suspected that the copper coil might exhibit induction in proportion as it was itself magnetized, and that thence the electricity might act instantaneously, as a magnet does when introduced into a coil [or helix]. I wished then to prove whether the copper might be unagnetized by the discharged electricities.

Having covered a copper cylinder with silk $I$ inclosed it in a coil, as I was accustomed to do with the iron cylinders. Having then placed it upon a magnetized needle, I caused some slight discharges of Leyden jars to pass through the coil itself, both weak and strong; but $I$ never had the smallest indication of magnetism in the copper.

IV. Having taken the said copper cylinder from the needle, I attached to its extremities two metallic wires, and these I connected with the ends of the wire of a re-electrometer; then discharging the Leyden jar by means of the coil which sur- 
rounded the cylinder, the re-electrometric deviations were no longer wanting.

I suspended the connexion between the little copper cylinder and the wire of the instrument, and having connected it instead with the ends of the coil which surrounded it, and then discharged the Leyden jars upon the cylinder itself, the usual deviations no longer failed. Are not these phænomena dependent upon Leyden-electrical actuation? As Iinvariably saw that, when I made the discharges pass through two points of the coil itself, there were very nearly the same deviations; and in the other case (namely, when the copper cylinder was connected with the wire of the re-electrometer) there was exactly the same effect when I discharged the jar connecting it with the two ends of the cylinder, I doubted whether, instead of inductions, I might not hitherto have seen only the effects of a division and subdivision of the discharge.

V. It appeared to me that I had taken the most scrupulous precautions that there might be no metallic contact between the actuating wire and that to be actuated; but might it not be that even through the silk the electricity might make itself a passage? The doubt was so much the more reasonable, as from the experiments related in the preceding memoir I had learned that the currents of the Leyden jars might divide themselves between good and bad conductors; I sought therefore to clear up this doubt by the following experiments.

I put the ends of the wire itself in metallic communication by means of a band of lead two centimeters broad and eight decimeters long; I covered one part with a small piece of very dry wool; then upon this wool and above the band itself I extended for the space of a decimeter, another band of the same metal, which rose at a right angle from both parts, for the space of four centimeters. Having many times discharged the Leyden jar, now in one manner, now in the other, putting the coatings in contact with the extremities of the second band, deviations of two, three, and even six degrees in the needle of the re-electrometer were always obtained.

I repeated the same experiments after having put four small pieces of wool between the band of lead adjoining the ends of the re-electrometric coil and that upon which the jars were discharged, and the results were pretty nearly the same.

The deviations were somewhat smaller (though they never failed) when, besides the said pieces of wool, $I$ also placed between the two metallic bands a large cake of sealing-wax having a strongly insulating power, and six good millimeters in size. And as in these experiments I made use of a small jar highly charged, and the spark passed thence to a great $2 \mathrm{~L} 2$ 
distance, so to remove my doubt that some part of the discharge might fall upon the neighbouring bodies instead of the extremity of the metallic band upon which $I$ intended the spark to pass, I attached two long copper wires to the extremities of the said band, and I went to some meters distance to discharge the jar; but the effects upon the re-electrometer were not different from those already observed.

If these experiments do not serve to show that the current of the Leyden jar passing through a metal causes in a neighbouring metal an electric current by induction, they would yet prove a very singular and unexpected property of the discharge of the Leyden jar; that I mean of dispersing itself in part in the worst conductors, even after having begun to traverse the best, since it must be said that the electric fluid descending by the vertical band scarcely reaches the point where this touches the wool or the sealing-wax, when a part of it quickly passes through the wool itself, and finding the band of lead underneath, a fraction of this part of the current passes into the band itself which is under the wool, and reaches the external coating of the jar by the shortest way; whilst the other fraction makes a much greater turn to traverse the coil around the re-electrometric iron, and at length reaching the wool from the opposite part, and there passing through the confining stratum, rejoins the other part of the larger band communicating with the external coating of the jar. That such a dispersion and division of discharge does not take place, seems proved by the experiments which I shall now describe.

VI. I made the experiments of the preceding section, but instead of laying the plate upon which the jar was discharged upon the confining portion for the space of two decimeters, I caused it to touch it only at a few points, bending it upwards at both sides; and I observed that if that part of the plate parallel to the under one was distant from the latter only a few centimeters, the effects upon the re-electrometer were still visible; but when the distance was two decimeters, there were no longer any deviations in the re-electrometer.

Having again laid the said plate upon the confining stratum, I cut in two the leaden band which communicated with the ends of the re-electrometer, and I placed near, between them, the sections, without their touching ; and this was done, because, if in discharging the Leyden jar a part of the current were carried upon this plate, crossing the confining part, all this portion must pass through the coil of the re-electrometer, and thence produce much greater deviations than when the band offered a continued conductor. But, on the contrary, the effects in this case were nothing; consequently, in these 
trials, either the suspected dispersion does not take place, or if it does, the movements of the magnetized needle do not proceed from it.

VII. Instead of placing the plate, which we will call the actuating plate, parallel to, and above the other which closes the re-electrometric circuit, and which we will call the actuated. one, I placed it parallel to it laterally, and at the horizontal distance of two centimeters. The currents produced by Leyden-electrical induction were somewhat weaker; but nevertheless sufficiently great to be seen, and measured.

VIII. Having replaced the actuating plate* above the other, and turned it somewhat in the horizontal plane round its central point, so that it might form an angle with the under one, the induced current was weaker, and still more so when the angle was greater. If the angle was of 60 degrees, the deviation caused in the instrument by the induced current was scarcely perceptible. And these experiments also prove, that the deviations do not proceed from a current transferred by dispersion, but rather from true Leyden-electrical induction.

IX. Having joined the ends of the re-electrometric coil by means of a fine copper wire, silvered, and then covered as usual with the little pieces of wool, and with the sealing-wax upon which was the usual plate of lead or copper, or zinc, upon which the jar was discharged, the induction took place as usual. It took place equally when the ends of the coil were connected with one of the said plates, and the jar was discharged upon the metallic wire duly placed upon the cake of sealing-wax.

$X$. I placed upon the said cake two insulating supports of glass, covered with sealing-wax, five centimeters high, and upon them the usual metallic plate, duly placed under one part of the re-electrometric wire. I then discharged the Leyden jar from one end to the other of the plate itself, and I had the deviation by actuation which at that distance I usually obtained.

Having inclosed in many folds of silk ribbin all but the extremities of the actuating plate upon which I wished to discharge the jar, and which I held in my hand parallel to the actuated plate, and carried to the height of six, ten, and even fifteen centimeters, the signs of electric induction never failed.

XI. The position and direction of the jar when discharging

* For the sake of brevity, I call a band or plate actuating through which the electricity passes, or ought to pass, its extremities being connected with the coatings of a Leyden jar or of a Franklinian square [see note, $p$. 509.]; and I call a plate actuated, in which (being near the former) arises, or may arise, an induced electric induction. 
it, never produced any difference in the results; certainly the effects were less when the distance between the two points of the plate upon which the jar was discharged was less : and if such distance was sufficiently small, as for example, one centimeter, there was no indication of an induced current, although the actuating plate might only be separated from the actuated by a very fine little portion of silk or wool.

XII. A band of lead, one meter long and two centimeters broad, was for the space of six decimeters inclosed in silk ribbin, leaving two decimeters of it bare at each extremity. I applied over this, but only for the space of five centimeters in the middle, a longer band of the same metal, and in order that they might be in better apposition to each other, I surrounded them both with silk thread in an open coil for all the said space. The uncovered band was then folded back, half a decimeter of the covered band remaining on each side to prevent the danger of any metallic contact between the two bands. I connected the extremities of the uncovered band with the metallic wire of a re-electrometer, and then discharged the Leyden jars, putting the two coatings in contact with the uncovered extremities of the band covered with silk ribbin, as above mentioned. During these experiments the currents produced by the Leyden-electrical induction caused much greater deviations in the magnetized needle than those which occurred in the experiments hitherto described, in which I caused the actuating current to act upon a part of the actuated band which was not longer than two decimeters.

The uncovered extremities of the leaden band partially covered with silk, having been connected with the wire of the re-electrometer, and the Leyden jar being discharged upon the extremities of the other band, the effects, in similar circumstances, were perfectly equal.

Again, with the weakest discharges and even with the first two or three residual charges, manifest indications of induction followed on experimenting with similar bands of lead, placed adjacent, in the manner I have mentioned.

XIII. Seeing that I could obtain induced currents by means of the weakest actuating currents, also by means of currents which when made to act directly upon the re-electrometer produced deviations less than those which followed from the actuated currents produced by strong discharges of Leyden jars, I thought it would not be time lost to attempt to obtain the inductions of the said induced currents.

A band covered with silk for the space of seven decimeters, with the extremities bare, had attached to all that part of it covered with silk, with the exception of two centimeters on each 
side, a second uncovered band; this remained on one side free for one decimeter; and on the other for nine, the two ends being connected together. Seven decimeters of this second band (in the space not attached to the first) were covered with silk, and to all this part, except two decimeters, was attached a third uncovered band, having also a space of one decimeter free on one side, and nine decimeters free on the other. Things being thus disposed, the ends of the first band were connected with those of the coil of the re-electrometer, and the ends of the third band were destined to be connected with the two coatings of the Leyden jar.

The trial being made, the deviations of the needle of the reelectrometer never failed. The usual small Leyden jar being charged as highly as possible, that is to about 80 degrees of the quadrant electrometer, there was a deviation of almost seven degrees. When charged to twenty degrees, there was a deviation of one degree and a half; and also with weak charges, and on many occasions with mere residual charges, I obtained visible deviations. Then the instantaneous current which passed into the third band of lead caused an induced current in the second, which formed as it were a large closed ring, and this current in its turn caused a second Leydenelectrical induction in the first band, which formed as it were a second large ring or closed circuit, being joined, as I have stated, to the re-electrometric coil.

I closed also the circuit formed by this third band, by connecting the ends of it, and covered a free space of seven decimeters with silk, and attached to it a fourth uncovered band in the manner now described; the Leyden jar being discharged upon this, there were unequivocal signs of an induction which we will call of the third order, and I thus obtained also the induced currents of the fourth and fifth orders, by adding another ring similar to those described, and then another still.

XIV. After the result here stated, there appeared to me no longer any doubt that the instantaneous currents of the confining armatures cause currents by true induction or actuation in the conductors near which they pass; and I addressed myself to study the properties of this action. I wished first to see whether the induction would take place even if there should be some metallic stratum as well as the confining strata between the actuating and actuated conductors.

Between two bands of lead, both covered with silk, I placed another, with care, in order that there might be no metallic contact between the bands. Having connected one of the covered bands with the wire of the re-electrometer, and dis- 
charged the Leyden jar upon the other similarly covered, the needle deviated, as when there was no naked band between the actuating and the actuated plates.

But if the two ends of the metallic band between the actuating and the actuated plates were connected, then the Leyden jar being discharged as above, there was no deviation in the re-electrometer, or it was very small, and only took place when the jar was considerably charged; which appears to me to prove, that when the actuating current operates upon a closed metallic circuit, it induces in it a contrary current, which either wholly or in part destroys the effect of the actuating current upon the second actuated band.

I connected the ends of the middle band with the re-electrometer, and now connected the ends of the third band with them, now employed them unconnected. In the first case, the jar being discharged upon the first band, the deviations were somewhat small, and in the second evidently greater. There was not so much difference in the two cases as in the preceding experiment, in spite of the small interval between the actuating band and the immediately actuated one.

XV. Two silvered copper wires were placed parallel to each other, each by means of two pegs of wood covered with sealing-wax, at one meter distant from each other, and moveable, so as to allow the distance between the wires to be varied, while they still remained parallel. Having connected one of these with the ends of the wire of a re-electrometer, and discharged the Leyden jar upon the other, I saw that the induction visibly took place, even when the distance between the actuating and actuated wires was seven centimeters.

I have already observed in $\$ X I$., that when the actuation only took place on a small space of the actuated conductor, the effect was less. Now, applying the ends of the re-electrometric wire to two points more or less distant from each other, I have observed that the signs of an induced current began to appear when the extent of wire subject to actuation was about a centimeter and a half, and the distance from the actuating wire two millimeters. When the actuated wire was three centimeters long a degree of deviation was obtained, and this increased to ten degrees when the extent of actuated wire was six or seven decimeters. It did not increase on the wire being considerably lengthened, which I tried to the extent of a meter. I have observed the same on varying the length of the actuating wire.

XVI. I connected the ends of a perfect Nobili's multiplier with those of the actuated metallic wire, and discharged the 
Leyden jar upon the actuating wire under the most favourable circumstances for obtaining the induced current; but the galvanometer did not make the smallest movement; nor was the result different when experimenting with the bands of lead instead of the copper wires; and this proves that such actuated currents are instantaneous, like the actuating.

XVII. On obliging the actuating current to pass through a metallic wire several hundred meters in length, the induced current was excited; nor did I perceive any different effect when it did not pass through that long wire. The same result also occurred when I forced the induced current to pass through the long wire before reaching the re-electrometer.

The induction did not fail either, when, instead of one end of the actuated wire being in metallic contact with one end of the coil of the re-electrometer, both were immersed in water, and distant from each other more than a decimeter; nor was the result different when the actuating current was made to pass through a stratum of water more or less thick.

The inductions of the second and third orders also, did not fail when made to pass through a long metallic wire or a stratum of water, although under such circumstances they appeared much weaker.

XVIII. Making use of a small iron cylinder surrounded by two parallel coils, similar to that mentioned in $\S$ I., I connected the two ends of one of them, and having discharged upon the other the small Leyden jar charged to fifteen degrees, no movement of the magnetized needle ensued; and the reason appears to me to be that the current induced in the circuit so formed was contrary, and nearly equal in magnetizing force to the immediate current.

I have also constantly observed, that the somewhat weaker currents of the first two or three residual charges were indicated by one or two degrees of deviation; and this, as it appears to me, arises from the weakest current not being sufficient to cause a sensible induced current in the wire circuit already mentioned.

Thus I have seen that a stronger charge, for example of forty or fifty degrees, was indicated by several degrees of deviation; never, however, so many as were observed when the other wire was unconnected: and this shows that when the wire was joined the immediate current prevailed over the induced.

XIX. The phænomena of induction of which $I$ have hitherto spoken, presented no anomalies to me; but this was not the case with respect to the direction of the induced current. In most of the experiments on this subject I made use of three 
small Leyden jars not having more than a square decimeter of exterior coating, and with each of these $I$ observed that the re-electrometric deviations indicated that the induced currents were directly contrary to the actuating. If, for example, the discharge of the jar in the direction of the band, or of the actuating wire parallel to the actuated, proceeded from right to left; in the band, or in the neighbouring actuated wire, the induced current passed from left to right: and, seeing in this an analogy to the volta-electric induction of Faraday*, I felt more and more persuaded that the phænomena observed proceeded really from Leyden-electrical induction. But I quickly began to doubt it when I applied myself to confirm, with the large jars, the results obtained with the small; for, on using these, the deviations of theinstrument indicated that the induced current, and that which caused it, proceeded in the same direction in the two parallel and neighbouring conductors.

I doubted whether, from the quantity of electricity being different in proportion to the tension, a different distance might not be required between the actuating and actuated conductors, in order to produce the direct current in a given manner in the latter; and whether in such phænomena there might not be something analogous to the inversions of magnetization observed by Savary in steel needles, placed at different distances from the conductors through which he made the discharges of great electrical batteries to pass. But whatever was the distance between the actuating and actuated conductors, which I have varied from one millimeter to a hundred, the inductions of the smaller jars were always directly opposite to those of the larger.

I turned my attention to the construction of the little jars, and I observed that they had the internal coatings formed of cuttings of tin-foil and silvered paper; I conjectured that the difference of effects in the large and small jars might depend on that discontinuity of the coating; and I long held this opinion, from having observed that two large jars, having the external coatings formed of so many small squares of tin-foil, of about one centimeter square, attached to the glass, so that between them might be a band of bare glass, of two or three millimeters broad, acted in precisely the same manner as the said small jars. But finally, having had some small jars prepared with internal and external coatings adhering to the glass, as in the large ones, I saw that they produced the currents by induction in the same direction as the large and small jars with discontinuous coatings; whence I was convinced that

[* See Faraday's'Experimental Researches in Electricity (26.), or Phil. Mag, Second Series, vol. xi. p. 300;-EDrr.] 
the cause of those different effects, although remote, depended on the different capacity of the jars ; for the large jars, with discontinuous external coatings, may be considered as furnished with small electric capacities only, as many of these small squares remain idle in charging or discharging the jars themselves.

Considering, then, that the least capacity of the confining coatings involves as a consequence, that with similar charges, that is, furnished with an equal quantity of electricity, the spark must pass to a greater distance, and thence find a similar expenditure in the longer space of air which it must traverse, I wished to see, if by effecting a retardation in the discharges of the large jars, there might perhaps be the same direction of the induced current that was observed with jars of less power.

I therefore caused the discharges of the large jars to pass through the water in a glass before reaching the actuating wire; and I saw that in this case these jars acted as those furnished with much less capacity. It is singular to see how the same quantity of electricity, put in motion by the same jar, induces a current, either in one direction or in that precisely opposite, according as it is, or is not, made to pass through a liquid stratum.

The different velocity, then, with which the electricity discharges itself from the one coating to the other, seems to give rise to the said inversions of phænomena: and in this opinion I was confirmed by having many times observed, when experimenting with the bands of lead described in $\$$ XII., that a jar of great power (capacita') strongly charged and discharged upon the actuating band, caused the induction contrary to that of the little jars; and with the first residual charge there was no effect, with the second and third an opposite one.

XX. A glass tube of about two centimeters in diameter, and twenty in length, was filled with spring water, and closed with two corks, through the axis of each of which passed a brass wire so far as to touch the water; both these wires projected out for the space of some centimeters, and terminated in a little globe. Having duly dried the exterior of the tube, and surrounded it with a band of lead two centimeters wide, which was twisted round it three times in the middle part of the tube, the ends of the bands were put in metallic communication with the extremities of the re-electrometric wire. Having discharged the Leyden jar so that it must pass through the water of the tube, I brought the external coating into contact with the little globe of one of the said wires, and the internal* with the little globe of the other, and the needle deviated two degrees.

[* Armatura externa in the original, but obviously in error.-EDIr.] 
Saline water being substituted for the spring water, a deviation of five degrees was obtained.

In order the better to assure myself of the insulation, I twisted round the tube a band of lead covered with silk; I repeated the experiments several times, and the results were always such as to lead to a conclusion that it was not necessary that the electricity should pass through a metal to cause the Leyden-electrical induction; it being sufficient that it should pass through some conductor, in order that the passage might be accomplished with sufficient celerity.

XXI. I connected the liquid contained in the tube which was used in the experiments above described, with the ends of the re-electrometric wire, and I then discharged the Leyden jar upon the extremities of the band which surrounded the tube itself. The needle deviated almost a degree. I renewed the experiment; but on repeating the same discharge in the same direction six times, I found the needle deviated four degrees. Hence we see that it is not necessary that the actuated conductor should be metallic, in order that the Leyden-electrical induction should take place.

From the experiments of this and the preceding paragraph, it may be deduced, that the induction would take place if neither the actuating nor the actuated conductor were metallic ; which, I believe, I have also verified by apposite experiments.

XXII. Having interrupted the actuating wire in another place, I connected one end of it with the external coating of a Leyden jar not charged, and the other with the internal $*$. I afterwards discharged a jar equal to that in power upon the wire itself. The induction took place, and the charge was divided between the two jars, which proves that it is not necessary that the identical fluid of one coating should pass to the other, to produce the phænomena of Leyden-electrical induction.

XXIII. Also the simple sparks drawn from the prime conductor may produce currents by actuation. Whilst one of the wires described in $\S \mathrm{XV}$. was connected with the re-electrometric coil, I let pass some sparks upon one extremity of the other, keeping the other extremity in communication with the ground; and I observed some movement in the magnetized needle at every spark that appeared.

Once, with fifteen sparks directed upon the actuating wire, a magnetization in the iron of the re-electrometer was obtained with the induced currents; which caused the needle to deviate three degrees.

[* Armatura externa in the original, but obviously in error.-EDrr.] 
But reserving to myself to treat, on another occasion, of the induced currents produced by sparks and by other electric currents, artificial or natural, it appears to me that we may in the mean time conclude,-

1st. That the instantaneous current of the Leyden jar, or of the Franklinian square* passing through a metallic current, causes an electric current, also instantaneous, in another metallic conductor, near to it, and forming a closed circuit,a phænomenon which I call Leyden-electrical induction, because analogous to that called by Faraday volta-electric induction.

2nd. That the same induced current may cause in another conductor a second current of induction; and this second, again another, and so on; whence may be produced currents of Leyden-electrical induction, of the second and third orders, \&c.

3rd. That the Leyden-electrical induction also takes place when the circuit through the metallic actuated conductor is closed by a very long metallic conductor, or even by a conductor not wholly metallic.

4th. That such induction also takes place when the discharge of the Leyden jar traverses a very long metallic conductor, and also a non-metallic conductor; nor does the phænomenon fail to appear, when it is not the identical fluid of one coating which passes to the other.

5th. That the induced current takes in the actuated conductor the same direction which the inducing current takes in the actuating conductor whenever the jar has great electric capacity, and is not too weakly charged. But the direction is opposite when the charge of the jar is rather weak, or when the electricity has to pass through a bad conductor, or when the jar is of small electric capacity.

6 th. That the phænomena of induction may be seen, although neither the actuating nor the actuated conductor is metallic.

7 th. That finally, such inductions are not exclusively from Leyden jars and the Franklinian square, but are obtained also with instantaneous electric currents from other sources.

[* Quadro Frankliniano ; meaning, we presume, the pane of glass with tin-foil conting on both sides; but this, we believe, was the device, not of Franklin, but of Smeaton.-EDIT.] 